\title{
Una Retrospección y Visión de Futuro sobre el Uso e Implementación de las Tecnologías de la Información y la Comunicación para el Aprendizaje Virtual en el Contexto de la División de Educología de la Universidad Nacional de Costa Rica
}

\author{
Gaby Ulate Solís \\ Enrique Vílchez Quesada \\ (Universidad Nacional de Costa Rica. Costa Rica)
}

\begin{abstract}
Resumen
La División de Educología de la Universidad Nacional de Costa Rica (UNA), ha sido consciente de la necesaria planificación y de un pensamiento en el futuro que demanda una reflexión continua entre lo posible y lo deseable. A este respecto, esta unidad académica inició desde hace varios años, procesos de investigación convergentes a la incorporación de las nuevas tecnologías en la docencia y sobre todo en la formación de los futuros educadores y educadoras del país. En este sentido, la formación de estos profesionales en sus distintas especialidades, busca fomentar no solamente el uso de la tecnología, sino también, el desarrollo de un juicio crítico y objetivo que abarque tanto sus ventajas como sus limitaciones en el noble ejercicio de la enseñanza y el aprendizaje. A la luz de estos preceptos, en el año 2007 emergió la actividad de investigación en docencia denominada Recursos Didácticos para el Aprendizaje bajo un enfoque bimodal. El presente artículo expone las ideas principales que se han madurado después de una experiencia de más de dos años con este curso, hacia la búsqueda de un re direccionamiento que retoma de manera responsable la planificación, implementación y las políticas de uso de TIC's (tecnologías de la información y comunicación) para el aprendizaje bimodal en el contexto de la División de Educología de la UNA.
\end{abstract}

\section{Palabras clave}

Enseñanza - Aprendizaje - Tecnologías - Recursos - Bimodal.

\section{Summary}

The Division of Educology of the National University of Costa Rica (UNA) has been aware of the necessary planning and of a thought in the future that demands a continuous reflection between the possible thing and the desirable thing. To this matter, 
this academic unit began for several years, to converge investigation processes to the incorporation of new technologies in teaching and mainly in the formation of the country's current and future educators. In this sense, the formation of these professionals in its different specialties, looks for to foment not only the use of the technology, but also, the development of a critical trial and objective that it embraces as much its advantages as its limitations in the noble exercise of teaching and learning. By the light of these precepts, in 2007 an investigation activity emerged in teaching Didactic Resources for Learning in a bimodal model. The article exposes the main ideas that have been matured after an experience of more than two years with this course, towards the search of a new direction that recaptures in a responsible way the planning, implementation and the policies of use of TIC's (Information and Communication Technologies) for the bimodal learning in the context of the UNA's Division of Educology.

\section{Key words}

Teaching - Learning - Technologies - Resources - Bimodal.

\section{Introducción}

En el contexto de la Universidad Nacional de Costa Rica emergió en el año 2006 la iniciativa de incorporar el uso de las tecnologías relacionadas con el aprendizaje virtual a distintos cursos ofrecidos por la División de Educología. La División de Educología es una unidad académica conformada por diversos profesionales que imparten los cursos de pedagogía a estudiantes de todas las carreras relacionadas con la enseñanza de distintas especialidades, como por ejemplo: Enseñanza de la Matemática, Enseñanza de las Ciencias, Enseñanza de los Estudios Sociales, Enseñanza de las Artes, entre otras (en total son catorce énfasis distintos). Educología pertenece al Centro de Investigación y Docencia en Educación de la UNA.

La División de Educología, ante los retos impuestos por el uso de las tecnologías y sus bondades y limitaciones en el ámbito educativo, creó un proyecto de investigación denominado "Docencia Virtual". En un inicio dentro de un marco institucional que desde el año 2004 ha venido impulsando una política de desarrollo donde las tecnologías ocupan un lugar preponderante en la pedagogía, el proyecto se centro en la capacitación de los docentes hacia el uso tecnológico de ciertas herramientas de carácter ofimático, quedando relegada la formación en la conceptualización y diseño de cursos en entornos de aprendizaje virtual como originalmente se había propuesto.

En el año 2007, en el marco de este proyecto de investigación, surgió otro denominado "Propuesta del curso DEX 325 Recursos Didácticos para el Aprendizaje bajo un enfoque bimodal, en las carreras de Enseñanza de la Matemática y Enseñanza de las Artes y Comunicación Visual", esta iniciativa contempló en un período de un año las etapas de planificación general, operati- 
va, ejecución y evaluación del curso "Recursos Didácticos para el Aprendizaje" con una modalidad que combinó la presenciabilidad con la virtualidad, lo cual resultó toda una innovación pedagógica dentro de la División de Educología por dos razones principales: en primer lugar los docentes y estudiantes en su mayoría no tenían formación académica y experiencias previas en el aprendizaje virtual como otra modalidad de estudio y en segundo lugar, en la División de Educología desde hace varios años ya se hacía necesario un curso que capacitara y formara a los estudiantes en el uso de ciertas tecnologías, como recursos didácticos dentro de sus distintas especialidades, además del uso de una plataforma de teleformación que en sí misma, se constituyó en un recurso didáctico para enseñar recursos didácticos, jun gran reto!, si se piensa en las características de una tradición pedagógica dentro de una universidad con una fuerte tendencia hacia lo presencial y el escaso uso de tecnologías para la enseñanza. Posiblemente dentro de otros contextos el proyecto no resultaría tan innovador como ha ocurrido en la UNA.

\section{Construyendo futuro}

Es importante reconocer que los esfuerzos realizados en la División de Educología de la Universidad Nacional de Costa Rica, parten de un pensamiento docente colectivo que mira hacia el futuro, que pretende construir para el futuro y que reconoce su responsabilidad al respecto como un grupo encargado de formar y sensibilizar a las nuevas generaciones interesadas en el sector educativo de nuestro país.

Pese a ello, las etapas de un proceso de planificación serio pecan por algunos de los inconvenientes suscitados en las transformaciones organizacionales que apuntan al cambio, muchas veces con poco tiempo de análisis ante sus limitaciones y posibilidades reales.

Los procesos de diagnóstico realizados en la División de Educología de la UNA, no han sido lo suficientemente estructurados como para prever las necesidades de infraestructura y de equipos informáticos actualizados, al servicio del cuerpo docente y las grandes cantidades de estudiantes que provienen de zonas rurales del país y que no cuentan con un computador en sus residencias temporales. Esto desde luego, está ocasionando graves problemas de acceso a los alumnos y docentes. Los alumnos se quejan constantemente de sus limitaciones económicas y pocas facilidades para realizar sus tareas virtuales y muchos profesores se disputan semana a semana el uso de los recursos disponibles, por ejemplo, el video bin o el único laboratorio de informática con el que cuenta la unidad, para atender más de cuarenta cursos semestre a semestre.

La prospectiva entendida como una anticipación para construir el futuro de manera planificada desde el presente (Prieto, 2008a) en la División de Educología, tiene un comportamiento disgregado, algunos docentes, cercanos a las políticas de la unidad, apoyan todos los procesos de innovación pedagógica a 
través del uso de las tecnologías y otros se resisten a su uso. Aunque este punto no preocupa tanto, pues en el ámbito educativo el convencimiento profesional es una tarea bipartita entre el tiempo y la capacitación docente.

La gestión académica de la unidad está derivando hacia una planificación general cuyos resultados convergen en la articulación de distintos proyectos de investigación, incluidos en áreas estratégicas de intereses macros (UNA) y micros (de la División propiamente). La evaluación de todos estos procesos se está realizando de forma desintegrada, a futuro inmediato es un aspecto fundamental a considerar dentro de la gestión general de la División de Educología.

\section{Sobre el curso Recursos Didácticos para el Aprendizaje}

En el año 2007 se inició el reto de incorporar dentro de la formación de futuros formadores, un curso donde los alumnos lograran comprender y aplicar el uso apropiado de distintos tipos de tecnologías tanto analógicas como digitales, en y para la docencia.

Lo interesante de esta experiencia ha consistido en el ambiente institucional desde donde la idea emergió, en una Universidad que por décadas ha sido predominantemente presencial y la docencia universitaria ha demostrado un claro énfasis en la experticia por los contenidos y no tanto en las prácticas pedagógicas de sus profesores. Este último aspecto ha llamado la atención de las autoridades universitarias actuales, proponiendo formalmente en su gestión un modelo pedagógico definido y reconocido organizacionalmente.

En este sentido, el curso Recursos Didácticos para el Aprendizaje bajo un enfoque bimodal, se propuso con el objetivo de iniciar el desarrollo de las competencias requeridas por los estudiantes en la aventura del uso experimental de recursos didácticos basados en tecnologías analógicas como la radio, la televisión y el retroproyector y tecnologías digitales tales como Internet y el uso de software (CmapTools, Photoshop y Dreamweaver), todo ello, con fines educativos.

El plan inició en el año 2007 específicamente en dos carreras; Enseñanza de la Matemática y Enseñanza de las Artes y Comunicación Visual y actualmente se ha hecho expansible a las catorce carreras a las cuáles se dirige. En el año 2008, los resultados no fueron tan favorables como los obtenidos en el 2007 y las razones se circunscriben en problemas clasificados en las dimensiones: pedagógica, tecnológica, de tradición institucional y de las características del sistema educativo nacional. Estas dimensiones fueron identificadas gracias a la aplicación de un instrumento a cuarenta y cinco estudiantes de las carreras Enseñanza de la Matemática, Enseñanza de las Artes y Comunicación Visual, Enseñanza del Inglés y Enseñanza del Español en el año 2008, además de otro instrumento dirigido a cada uno de los docentes responsables de estos cursos. 


\section{Problemas a resolver}

Justamente en el contexto expuesto, ha surgido un problema pragmático muy claro; continuar el curso Recursos Didácticos para el Aprendizaje bajo un enfoque bimodal, o volver a impartir el curso con sus características netamente presenciales, sin haber dado cabida a generalizar esta experiencia sistemáticamente a otros cursos que se imparten en la División de Educología de la UNA.

El problema trasluce elementos de mayor complejidad, creemos y demostramos en el año 2007 (Vílchez y Ulate, 2008) que el curso fortalece las competencias de los alumnos en cuanto al uso de recursos didácticos tradicionales (analógicos) e innovadores (digitales), además de mejorar sus condicionamientos cognitivos generando oportunidades para favorecer el autoaprendizaje y el coaprendizaje, sin embargo, ¿por qué en el año 2008 los resultados no fueron los esperados?, tenemos algunas hipótesis que nos han permitido estructurar una visión de futuro sobre el uso e implementación de las TIC's para el aprendizaje virtual en la unidad académica y que expondremos en el presente trabajo. Consideramos que el curso Recursos Didácticos para el Aprendizaje bajo un enfoque bimodal ofrece verdaderamente los espacios de enseñanza y aprendizaje descritos (y así podría ocurrir con otros cursos), pero para ello, el perfil docente, la propuesta pedagógica, los insumos tecnológicos ofrecidos por la institución a profesores y estudiantes, la normativa que rige la implementación de estas modalidades, la resistencia al cambio y la herencia cultural de un sistema educativo predominantemente presencial y positivista, requieren de un análisis de construcción de futuro que albergue los retos y alternativas de solución enmarcados no sólo en la coordinación del curso, sino también, en la unidad académica en su conjunto y en la Universidad en general, hacer explícitos estos aspectos es el principal objetivo del presente artículo.

\section{Justificación del problema}

Construir futuro en la División de Educología de la Universidad Nacional de Costa Rica no significa ir en concordancia ciega con las nuevas o relativamente nuevas tendencias hacia el uso de las tecnologías digitales, significa pensar y repensar la manera en como ellas pueden impactar en el futuro de la educación costarricense en general.

Buscamos soluciones en el mediano y largo plazo que tengan una repercusión en las prácticas educativas de los docentes y por tanto en las prácticas de aprendizaje de los estudiantes. Con frecuencia llamamos a nuestros actuales educandos "nativos digitales", pero qué significa realmente ser un nativo digital, bastará con haber crecido presionando botones y conviviendo con pantallas, pareciera que ésta es la convicción irracional de muchos, ciertamente en esta época confusa nos encontramos con "salvajes digitales" " "ilustrados digitales", 
¿qué estamos formando?, ¿somos conscientes en las universidades de lo que queremos y la dirección contextual que deben llevar las tecnologías hacia la estructuración de una sociedad conformada por personas competitivas, críticas y altruistas? Creemos que los pasos de transformación de esta utopía a un escenario real inicia con acciones concretas, aventureras pero no descabelladas, ambiciosas pero no descontextualizadas, en el marco de lo idealizable pero no de lo desproporcionado. A esto, precisamente, aspira responder el proyecto del curso Recursos Didácticos para el Aprendizaje bajo un enfoque bimodal, hay razones muy puntuales ya mencionadas en el apartado anterior, para justificar la continuidad de este trabajo dada su importancia y aportes institucionales. Sin embargo, ante las dificultades y las limitaciones presentadas en el año 2008, y la necesidad de no desperdiciar los recursos económicos y humanos de la UNA, es fundamental hacer un alto en el camino, no para negar la responsabilidad ya adquirida, sino para establecer un replanteamiento de esta cátedra y en general de las políticas de uso e implementación de TIC's en la División de Educología de la UNA, que apoyan los procesos de enseñanza y aprendizaje bimodales. Nos encontramos ante un océano de oportunidades que con el tiempo creemos convergerán evolutivamente a una oferta de servicios, en temas relacionados con la calidad de la docencia semipresencial en Costa Rica.

\section{Contexto del problema}

La Universidad Nacional constituye una de las cuatro universidades públicas de Costa Rica, con una oferta educativa basada en la presenciabilidad desde su fundación. En la actualidad el modelo pedagógico en el que se sustenta se fundamenta en los siguientes principios:

- Respeto a la diversidad en todas sus expresiones.

- Respeto y compromiso con la igualdad de oportunidades y con la construcción de una sociedad más justa y equitativa.

- Formación de profesionales solidarios y comprometidos con el bienestar social.

- Flexibilidad para conceptuar el aprendizaje como proceso sociocultural, histórico, dinámico y transformable, posible y que puede construirse de muchas maneras.

- Interacción en los procesos formativos donde los conocimientos sean discutidos y enriquecidos permanentemente.

- Formación de un espíritu investigador en los futuros profesionales.

- Creatividad que permita la innovación, así como la utilización de medios, estrategias y recursos de enseñanza en los procesos de mediación pedagógica.

- Disposición para determinar los principios lógicos subyacentes en cada disciplina, que permitan una formación profesional de calidad. 
- Evaluación como proceso integral, concertado, permanente, contextualizado y propositivo.

- Mejoramiento continuo en la formación integral de los estudiantes y los procesos de gestión académica-administrativa y paraacadémica.

- Visión prospectiva que permite la planificación estratégica para el logro de objetivos a mediano y largo plazo.

Estos principios fueron extraídos del modelo pedagógico institucional publicado en la dirección www.una.ac.cr, que corresponde al sitio oficial de la UNA.

Del modelo principalmente es rescatable el compromiso organizacional que asumen todos los docentes hacia una cultura de planificación prospectiva, que permita a partir de la adaptación de sus estrategias de enseñanza y aprendizaje, alcanzar logros que a mediano y largo plazo posibiliten la innovación, el enriquecimiento de los contenidos y la utilización de medios y recursos creativos en la mediación pedagógica. ¡La institución reclama cambios!, invita a modernizar de manera contextual las prácticas educativas cotidianas sea en el ámbito presencial o semipresencial.

En particular la División de Educología de la UNA, en consistencia con el modelo pedagógico anterior, se ha propuesto los siguientes objetivos:

- Contribuir a la transformación de la sociedad costarricense hacia un desarrollo integral, autónomo y sostenible, formando profesionales críticos, independientes, participativos y creativos con un dominio sólido del conocimiento de su disciplina y su problemática pedagógica.

- Formar integralmente educadores para la enseñanza, desde un currículum interdisciplinario que articule la educación con otros campos del conocimiento y fortalezca la identidad profesional de los docentes.

- Crear ambientes académicos favorables a la construcción y reconstrucción del conocimiento acerca de los procesos de enseñanza-aprendizaje de una disciplina y de sus vínculos con los procesos educativos en los que ocurren.

- Formar profesionales en educación con sentido de pertenencia cultural, a partir de principios y valores democráticos, que incluyen la tolerancia, la equidad, el respeto por las diferencias, por la vida en todas sus formas, su ambiente y el derecho a la felicidad.

- Desarrollar la vocación investigadora en los educadores y educadoras para que fortalezcan los procesos de enseñanza-aprendizaje y los vinculen con la realidad educativa en la que trabajan.

La información anterior está disponible en: http://www.una.ac.cr/cide/ Educologia/index.html.

Propiamente en el ámbito del uso de los recursos didácticos, la unidad da una especial importancia a la vinculación de las Ciencias de la Educación 
con otras áreas de conocimiento, específicamente el área de las tecnologías analógicas y digitales en cuanto al impacto que producen en el sector educativo, con sus limitaciones y sus fortalezas. A este respecto, el curso Recursos Didácticos para el Aprendizaje representa bajo su modalidad semipresencial, una respuesta ante los retos impuestos no solamente por la nueva generación de jóvenes que ingresan a la UNA y a carreras relacionadas con la enseñanza, sino también, una respuesta en el seno de una institución que tanto de forma macro como micro reconoce el valor y la importancia en la formación para el uso didáctico de los distintos recursos disponibles de mediación pedagógica en correspondencia crítica con las nuevas tendencias.

La UNA, además, el 30 de agosto del 2008 aprobó el documento denominado "Políticas para la incorporación de las tecnologías de información y la comunicación en los procesos académicos de la Universidad Nacional" que para bien o para mal está regulando la incorporación de las tecnologías de información y comunicación en los procesos académicos institucionales, por medio de una entidad centralizada llamada UNA Virtual, quien es la responsable de asesorar, promover y desarrollar iniciativas de incorporación de las TIC's en el quehacer académico, además de administrar y desarrollar el Aula Virtual de toda la universidad implementada de manera oficial en la plataforma Moodle. Los resultados no han sido los mejores pues la centralización de servicios con una alta demanda ha ocasionado ruptura comunicacional, lentitud en la resolución de problemas, lentitud en la apertura de los cursos y poco codesarrollo de la plataforma Moodle ante las necesidades particulares de la UNA y sus distintas Escuelas. Por otra parte, vislumbramos a futuro con mucho temor, una burocratización de los procedimientos para la innovación, pues si una unidad académica decide utilizar cierto tipo de TIC's en sus procesos educativos, ha perdido parte de su hegemonía y libertad académica teniendo la obligación de hacer consulta primero al departamento de UNA Virtual, ajeno y en muchos casos desconocedor de las necesidades particulares. ¡Bien o mal este es nuestro marco jurídico!

La disponibilidad de laboratorios y licenciamiento de software, es otro de los aspectos que preocupan a nivel organizacional. Ya existe un grupo de académicos denominado UNA Alternativa de distintas Escuelas que están promoviendo el uso de software libre como solución ante las problemáticas emanadas por el software privativo y con una fuerte influencia provocada por la tendencia mundial que ha traído este movimiento en muchos países, como por ejemplo España. Pese a los esfuerzos aún estamos en camino y en una edad temprana. En la División de Educología de la UNA, además, los recursos son pocos, tanto los audiovisuales como de acceso al único laboratorio disponible para todos los cursos que se imparten semestre a semestre, un detalle operativo muy importante por las exigencias de acceso que demanda una modalidad híbrida. 
Como parte de este ejercicio de diagnóstico, la caracterización de los alumnos que ingresan a las carreras relacionadas con la enseñanza en la Universidad Nacional de Costa Rica, se resume de manera genérica en los siguientes puntos:

- Muchos provienen de zonas rurales en las cuales durante su educación primaria y secundaria tuvieron poco contacto con el uso de tecnologías digitales, inclusive, muchos entran a la universidad sin saber cómo crear siquiera una cuenta de correo electrónico.

- Los estudiantes se muestran relativamente neófitos en actividades relacionadas con la lectoescritura y hábitos de investigación.

- Los alumnos prefieren en su mayoría las clases presenciales y magistrales, presentando una fuerte resistencia a la modalidad virtual.

- Los estudiantes no saben trabajar de manera colaborativa, ni cooperativa, aunque están acostumbrados a relacionarse de forma grupal, dividiéndose tareas y unificando regularmente sus resultados.

¡Y bien!, con este panorama general descrito, iniciaremos un análisis de un escenario que hemos venido idealizado para cualquier curso semipresencial en la División de Educología de la UNA, desde hace dos años con la implementación del proyecto Recursos Didácticos para el Aprendizaje bajo un enfoque bimodal y realizaremos un ejercicio retrospectivo contrastando la experiencia acumulada hasta la fecha y las características institucionales.

\section{Futuro esperado}

El proyecto Recursos Didácticos para el Aprendizaje bajo un enfoque bimodal, emergió con la idea de institucionalizar un curso de pregrado dentro de la formación de futuros educadores, en una modalidad blended-learning que lograra aproximar a los estudiantes a otras realidades educativas concordantes con el uso de las tecnologías de la información y la comunicación. Durante toda la experiencia desarrollada en torno al proyecto, hemos consolidado un escenario ideal que debería orientar cualquier iniciativa relacionada con el aprendizaje virtual dentro de la División de Educología de la UNA. Este escenario se circunscribe en los siguientes aspectos:

- En la dimensión pedagógica: los docentes presentan una formación y experiencia adecuada en el diseño y manejo de un entorno de aprendizaje virtual, además son conscientes de las diferencias y similitudes entre los espacios educativos presenciales y virtuales. Se sienten comprometidos con una modalidad educativa bimodal, reconociendo el aporte que ésta puede proporcionar en cuanto al desarrollo de competencias que sin el uso de esta modalidad, sería más difícil integrar en la formación de los estudiantes. Los docentes presentan las habilidades necesarias para la elaboración de material didáctico dirigido a este tipo de escenarios edu- 
cativos. Finalmente, los profesores tienen conocimiento sobre estándares de calidad en la educación a distancia.

- En la dimensión tecnológica: los profesores utilizan la tecnología con una posición pedagógicamente crítica, son usuarios pero también codesarrollares, definiendo con claridad sus necesidades y los aspectos en los cuales la plataforma Moodle de la UNA debería converger con ayuda del grupo UNA-Virtual. Los docentes tienen formación tanto en el uso técnico de las tecnologías analógicas como también de las digitales. Poseen conocimiento de experiencias internacionales sobre el uso de estas tecnologías en el sector educativo. Son usuarios activos de la Red y las tecnologías Web 2.0, reconociendo desde su visión de consumo sus ventajas y limitaciones.

- En la tradición institucional: la Universidad Nacional de Costa Rica reconoce la importancia y necesidad de crear espacios educativos semipresenciales y virtuales, funda un Centro de Materiales Audiovisuales que apoyan los procesos de enseñanza y aprendizaje a distancia. En su normativa institucional, se brinda una atención particular a las modalidades semipresenciales y virtuales, en cuanto al reglamento de evaluación de los aprendizajes y a los derechos y deberes tanto de los académicos como de los estudiantes. Institucionalmente se brinda a los distintos grupos interdisciplinarios el tiempo necesario dentro de su jornada, para la conceptualización, diseño e implementación de cursos y programas bajo estas modalidades.

- $\quad$ En el sistema educativo nacional: el Ministerio de Educación Pública, con apoyo de los académicos de la División de Educología de la Universidad Nacional, inicia procesos de formación y capacitación docente en tutoría virtual, entornos de aprendizaje virtuales y capacitación específica en didáctica, pedagogía y antropología.

Por otra parte, vislumbrando el escenario posible en cada una de estas dimensiones, pretendemos en un plazo máximo de cinco años obtener los siguientes logros:

- En la dimensión pedagógica: docentes capacitados en el área de la tutoría virtual, al menos con un curso aprobado por una organización internacional. Hay claridad sobre el perfil de los profesores que constituyen las distintas cátedras de los cursos que se imparten de forma semipresencial, lo cual proporcionará insumos importantes de contratación de personal. Se consolidará un grupo de tres o cuatro investigadores en el área para determinar en el contexto de la UNA de Costa Rica ventajas, desventajas y necesidades ante las exigencias de una modalidad blended-learning. Se contará con un grupo de tres a cuatro profesionales que de manera permanente, se dedicarán a elaborar y reelaborar materiales didácticos para el curso Recursos Didácticos para el Aprendizaje u otros que se impartan de forma bimodal. 
- En la dimensión tecnológica: los docentes que formarán parte de las cátedras de cursos semipresenciales, se capacitarán en el uso de distintos recursos tecnológicos tanto analógicos como digitales, recibirán el estímulo de contar con una beca semestral para participar de un curso en el Instituto de Capacitación y Asesoría Informática, o bien, en el programa Identidad Cultural, Arte y Tecnología ambos centros de la Universidad Nacional de Costa Rica. Anualmente la coordinación general de las cátedras de los cursos bimodales, recogerá información para detectar las debilidades y fortalezas de la plataforma Moodle implementada en la UNA, esta información retroalimentará los esfuerzos del departamento UNA-Virtual para robustecer y adecuar la plataforma institucional. Finalmente un grupo de dos o tres especialistas, actualizará a todas las cátedras con experiencias internacionales que alimenten el quehacer académico en el contexto de todos los cursos semipresenciales.

- En la tradición institucional: se solicitará a la Dirección de la Unidad Académica un pronunciamiento anual si es necesario, de parte del Consejo de Unidad y Consejo de Facultad a la rectoría, para regularizar en el marco jurídico los derechos y obligaciones de académicos y estudiantes en el contexto de una modalidad semipresencial y/o virtual.

- En el sistema educativo nacional: la División de Educología ofrecerá prestación de servicios a la comunidad costarricense en general, para la implementación exitosa de cursos bimodales o virtuales. El proceso iniciará sin ningún costo ofreciendo asesoría al Ministerio de Educación Pública en temas relacionados con la tutoría virtual y la complejidad de los entornos de aprendizaje virtual.

La propuesta pedagógica macro de los cursos con características virtuales y sus objetivos bajo la modalidad blended-learning, se presentan en los siguientes apartados.

\section{Propuesta pedagógica}

En los cursos de la División de Educología bajo un enfoque bimodal, el uso, construcción y aplicaciones en el ámbito educativo de diversos recursos didácticos tanto analógicos como digitales, se fundamenta en el reconocimiento del aprendizaje como un producto social de acuerdo con Vygotsky (Espiro, 2008d) y como un proceso activo según Ausubel y Bruner (Espiro, 2008d) a partir del cual los alumnos construyen o reconstruyen ideas, conceptos, soluciones, estrategias, conjeturas y aplicaciones de la teoría a situaciones reales o hipotéticas en los diversos contextos educativos que caracterizan al sistema educativo nacional.

Se fomenta la construcción social del conocimiento mediante el trabajo en equipo, cooperativo y colaborativo. Se espera que los alumnos se apropien 
de habilidades y destrezas de investigación, que les permita tener una mayor independencia sobre sus propias estructuras de pensamiento, aprendizaje de estrategias de aprendizaje (aprender a aprender) y el desarrollo de un pensamiento crítico a través del ejercicio dialógico, el respeto por los saberes individuales y colectivos, y la creación conjunta de nuevas ideas, tal y como nos lo plantea la educación liberadora propuesta por Paulo Freire (Espiro, 2008e).

El andamiaje citado por Bruner y el aprendizaje significativo desarrollado por Ausubel, en muchas de las estrategias didácticas que caracterizan a los cursos, cobran una posición central tanto en el ejercicio de la tutoría virtual y presencial, como en las actitudes que se espera desarrollar en los educandos. El docente asumirá el reto de elaborar y seleccionar materiales de apoyo (lecturas, presentaciones, link, recursos de la Web 2.0, problemas) que inciten el descubrimiento de técnicas y métodos donde el alumno encuentre los espacios necesarios para una construcción y aplicación personal y colectiva de los conocimientos relacionados con cada unidad temática.

Finalmente, esta propuesta pedagógica toma en consideración también, la importancia del aprendizaje receptivo y el condicionamiento operante propuesto por Skinner (Espiro, 2008d), en el ejercicio de la tutoría virtual estimulando la práctica de una conducta social adecuada y los fundamentos de una comunicación asertiva, respetuosa y tolerante, utilizando las distintas herramientas de comunicación tanto sincrónicas como asincrónicas del aula virtual. Además, se reconoce el valor del aprendizaje memorístico como puente para conciliar los conocimientos teóricos previos, con estrategias de enseñanza no mecanicistas.

\section{Objetivos}

El objetivo general que se ha dispuesto en la visión de futuro sobre el uso e implementación de las TIC's para el aprendizaje virtual en el contexto de la División de Educología de la Universidad Nacional de Costa Rica, es el siguiente: desarrollar procesos formativos en los educandos hacia el uso, construcción y aplicación de las distintas unidades temáticas de los cursos, a través de una construcción individual y social del conocimiento que promueva una conciencia pedagógica crítica y constructiva, de las aplicaciones y limitaciones que tienen las tecnologías analógicas y digitales en el ámbito educativo.

Como objetivos específicos proponemos los siguientes:

- Utilizar distintos tipos de tecnologías analógicas y digitales como apoyo en los procesos de enseñanza y aprendizaje.

- Identificar ventajas y desventajas que aportan las tecnologías analógicas y digitales en los diversos contextos de acuerdo con el área cognitiva de cada disciplina y área en estudio. 
- Identificar fortalezas y debilidades de uso de los distintos tipos de recursos didácticos que aportan las tecnologías analógicas y digitales al sistema educativo costarricense.

\section{Resultados esperados}

Los resultados que se espera obtener con las distintas cátedras de los cursos que se circunscriban bajo un enfoque bimodal, se centran en los agentes principales involucrados con sus procesos de implementación: los estudiantes, los docentes, el departamento de UNA Virtual y la institución en general.

Los resultados esperados en un período de tiempo de tres años son los siguientes:

- La existencia de un coordinador general de todos los cursos tanto en sus características presenciales como virtuales, un grupo de dos profesionales con una carga de un cuarto de tiempo cada uno destinada a la investigación de las implicaciones teóricas que emergen en la práctica educativa de los distintos cursos, un experto de contenido y un diseñador gráfico para la elaboración y reelaboración de materiales didácticos con una carga académica de al menos un cuarto de tiempo.

- Un equipo de cinco docentes con formación académica debidamente acreditada en el área de la tutoría virtual. Se espera que este grupo de profesores se encuentren capacitados al menos con dos cursos relacionados con la tutoría virtual y el manejo de entornos virtuales de aprendizaje.

- Un profesional del departamento de UNA Virtual integrado debidamente con el proyecto y su coordinador general, para el manejo administrativo de la plataforma y en la oferta de servicios de capacitación y consultas técnicas tanto a profesores como a estudiantes, relacionados con el uso pedagógico y técnico de la plataforma Moodle.

- La publicación de un artículo anual en una revista científica internacional sobre los avances teóricos suscritos a las prácticas educativas en torno a los distintos cursos bimodales, los alumnos, la plataforma de teleformación utilizada y las prácticas docentes presenciales y virtuales más adecuadas en el contexto de la Universidad Nacional de Costa Rica.

- Una mediación pedagógica de todos los materiales y recursos didácticos utilizados en las diferentes cátedras de los cursos tanto para el trabajo presencial como virtual.

- Capacitación continua de los docentes de las cátedras en el uso de distintos tipos de recursos didácticos tanto analógicos como digitales. Se pretende que cada docente se capacite con al menos un curso anual.

- Se promoverán acciones para crear una conciencia institucional sobre la necesidad de incorporar y diferenciar en el marco jurídico de la Universidad, 
los derechos y obligaciones de académicos y estudiantes en el contexto de una modalidad semipresencial y/o virtual.

- Una propuesta de cursos madura y validada en: prácticas de aprendizaje, estructura y estrategias de evaluación que permitan migrar la experiencia a otros cursos de la División de Educología y a futuro ofrecer servicios de formación al Ministerio de Educación Pública de Costa Rica en esta materia.

\section{Aspectos operativos}

Los aspectos operativos relacionados con las políticas de uso e implementación de TIC's para el aprendizaje virtual en la División de Educología de la UNA, se especifican en este apartado, la exposición se divide en: prácticas de aprendizaje y tecnologías, materiales didácticos, tutoría prevista, administración del sistema, evaluación y seguimiento.

\section{Prácticas de aprendizaje y tecnologías}

En los cursos de la División de Educología que utilicen un enfoque blendedlearning, el trabajo se realizará mediante la acción conjunta de profesor(a) y estudiantes, en donde se planteen y desarrollen mediante la investigación y trabajo cooperativo/colaborativo, los diferentes temas que interesa conocer. El profesor(a) cumple una función de facilitador en la construcción del conocimiento, los estudiantes son los responsables de llevar a buen término su proceso de aprendizaje a través de las actividades propuestas.

Es esencial el trabajo en equipo, la búsqueda de consenso, lluvia de ideas para obtener significados, la búsqueda de información, la elaboración de categorías y los procesos de análisis y síntesis en la adquisición y reconstrucción del conocimiento.

\section{Materiales didácticos}

Los materiales de estudio que se utilizarán en los distintos cursos bimodales dentro de la División de Educología, serán seleccionados y/o elaborados, editados y adaptados por el coordinador general y de cátedra de cada curso, mediante la colaboración de un diseñador gráfico y los docentes-tutores. Los documentos o lecturas tendrán formato PDF, dada su facilidad de acceso y optimización en el peso de los archivos.

\section{Tutoría prevista}

El grupo de profesionales que se encargarán de brindar acompañamiento a los alumnos durante las dieciocho semanas de los distintos cursos bimodales, 
estará conformado por tutores debidamente capacitados en el área, quienes asumirán la responsabilidad de un grupo con a lo sumo treinta estudiantes.

Existirá la figura de un tutor general (coordinador) quien tendrá asignadas las siguientes funciones: velar por la calidad de los servicios académicos y administrativos ofrecidos a los estudiantes, proporcionar apoyo a los docentes (sobre todo a aquellos que se inician introduciéndolos al enfoque pedagógico de los cursos), coordinar con la persona encargada en el departamento de UNA Virtual la apertura de cursos y procesos de capacitación y encauzar las tareas de investigación en torno a este proyecto. Además, el coordinador general identificará los puntos débiles en cuanto a contenido, dominio tecnológico y tutoría virtual de su grupo de colaboradores y gestionará ante las autoridades de la unidad académica el presupuesto requerido, con el objetivo de capacitar a los docentes en las áreas prioritarias.

\section{Administración del sistema}

En el contexto de la Universidad Nacional de Costa Rica el departamento de UNA Virtual oficialmente tiene a su cargo la administración tecnológica de la plataforma institucional, en este caso y hasta la fecha, la plataforma Moodle. Bajo esta perspectiva, compete a este organismo la apertura de los cursos en toda la Universidad; sin embargo, en muchas ocasiones se ha comprobado en la práctica que esto tiene repercusiones importantes en el tiempo de creación espacial de cursos virtuales o semipresenciales. En este sentido, el coordinador general del proyecto velará por mantener una comunicación constante con el representante del departamento UNA Virtual a cargo, tratando de prever inconvenientes que pongan en riesgo el inicio exitoso de los cursos en sus componentes virtuales.

Los materiales de los cursos serán entregados a los estudiantes en un $\mathrm{CD}$ durante las dos primeras semanas del período lectivo, que se dictarán de manera presencial.

El coordinador general se encargará también de gestionar los procesos para evaluar la calidad de los cursos ofrecidos en cuanto a: prácticas de aprendizaje, estrategias de evaluación, actualización de los contenidos, bibliografía y lincografía, calidad de la tutoría, fortalezas y debilidades en la plataforma de teleformación, acceso a los laboratorios institucionales y rendimiento académico de los estudiantes.

\section{Evaluación}

La evaluación de las distintas cátedras en la División de Educología que utilicen un enfoque bimodal, integrará indicadores de calidad distribuidos en los siguientes aspectos: la gestión de los cursos, los materiales didácticos, los medios y tecnologías seleccionadas, la evaluación de los aprendizajes y 
la infraestructura que dará soporte a la implementación. Los indicadores han sido tomados como puntos de referencia del documento "Informe final" de la CONEAU (Becerra y Bavio, s. f.) y han sido adaptados a las características del proyecto que aquí nos ocupa, en el marco de acción de la División de Educología de la Universidad Nacional de Costa Rica. Las líneas siguientes explican el contexto particular en el cuál será imprescindible efectuar los procesos de autoevaluación en las dimensiones anteriormente establecidas.

\section{Gestión de los cursos}

Los cursos bajo un enfoque blended-learning como en algún momento ya fue explicado, contarán con un coordinador general. Con el objetivo de garantizar el buen funcionamiento de su gestión académica y administrativa, será indispensable que la persona más próxima de acuerdo a la jerarquía organizacional, realice una evaluación sobre el cumplimiento de sus principales funciones, las cuales se pueden resumir en:

- Cuidar la calidad de los servicios académicos y administrativos ofrecidos a los estudiantes.

- Brindar apoyo a los docentes en asuntos tanto académicos como administrativos.

- Realizar procesos de inducción para aquellos profesores que se inician con el enfoque pedagógico de los cursos.

- Coordinar con la persona encargada en el departamento de UNA Virtual la apertura de cursos y procesos de capacitación.

- Analizar las fortalezas y debilidades de la plataforma institucional.

- Liderar los procesos de investigación en torno al proyecto.

- Liderar los procesos de mejoramiento de las cátedras en cuanto a contenido, prácticas de aprendizaje, experiencias de evaluación, dominio tecnológico y tutoría virtual.

- Gestionar ante las autoridades de la unidad académica el presupuesto requerido con el objetivo de capacitar a los docentes en las áreas prioritarias.

La dirección de la División de Educología será la responsable de velar por el cumplimiento de estas tareas, en la figura del coordinador general.

\section{Materiales didácticos}

Los materiales didácticos seleccionados y elaborados para las cátedras de los cursos, serán el resultado de un proceso continuo y colaborativo, por parte de los docentes/tutores quienes tendrán la responsabilidad de elaborar y/o adaptar estos recursos a sus distintas especialidades y/o áreas. En conjunto se diseñarán guías didácticas que integrarán todos los materiales por área tanto 
generales como específicos de las distintas disciplinas, estas guías servirán como hilo conductor de contenido y experiencias de aprendizaje para futuras promociones de los cursos. Con todo ello el proceso de evaluación de estos materiales a la luz de la percepción de los estudiantes deberá contemplar los siguientes aspectos:

- Los materiales son apropiados para la educación a distancia.

- Los materiales son claros y tienen una relación directa con los objetivos de aprendizaje de los cursos.

- Los materiales hacen referencia a aplicaciones concretas de los temas de los cursos a la disciplina en estudio.

- Tienen una presentación que incita a su lectura.

- Ofrecen información completa pero no acabada, mostrando al lector otras fuentes de información a materiales complementarios.

Los docentes que conforman las distintas cátedras tendrán un lugar preponderante en los procesos de autoevaluación dirigidos a mejorar la calidad de los materiales que caracterizan a los cursos, por tanto, el coordinador general al finalizar cada período lectivo, asumirá la responsabilidad de convocar a todos sus colaboradores para analizar los resultados obtenidos en las evaluaciones de la percepción estudiantil e iniciar las acciones que en conjunto conducirán a una reelaboración y/o re selección de los materiales.

\section{Medios y tecnologías}

En las cátedras de los cursos bimodales de la División de Educología de la UNA, los docentes asumirán la responsabilidad de analizar en conjunto con el coordinador general, el impacto que tienen en los procesos de enseñanza y aprendizaje las prácticas de aprendizaje implementadas en cada período lectivo.

Este aspecto será esencial para decidir cuáles tecnologías constituirán el soporte en la mediación pedagógica de las experiencias que conducirán a los educandos hacia el aprendizaje tanto individual como cooperativo. En este contexto, la observación de los registros dejados por los estudiantes en el aula virtual (en sus distintas asignaciones) se convertirá en el insumo de información básica para respaldar o refutar las decisiones tomadas por el grupo docente hacia el uso de otras tecnologías y medios que mejoren los resultados esperados en cuanto al desarrollo de habilidades meta cognitivas y de trabajo colaborativo.

Además, el coordinador general del curso se encargará también de valorar el desempeño de los docentes en cuanto a las habilidades tecnológicas demostradas durante y al finalizar el período lectivo, encausando las fuerzas administrativas necesarias para facilitar espacios de capacitación en primera 
instancia por medio de la colaboración del departamento de UNA Virtual y, más a largo plazo, mediante la inscripción en cursos especializados.

Finalmente, se aplicará un instrumento de evaluación a los estudiantes y docentes participantes de los cursos en sus distintas áreas y especialidades, para valorar las ventajas y limitaciones de la plataforma Moodle con sus características de instalación e implementación institucionales. La información suministrada y analizada por el coordinador general, brindará información precisa al departamento de UNA Virtual sobre las necesidades y fortalezas de la plataforma, con el objetivo de retroalimentar su trabajo hacia la búsqueda de una herramienta tecnológica que responda a las demandas sentidas por la comunidad universitaria.

\section{Evaluación de los aprendizajes}

Éste es uno de los aspectos más importantes en el contexto del proyecto aquí expuesto. Creemos como ya se ha manifestado, que los cursos basados en un enfoque bimodal, pueden potenciar el desarrollo de habilidades y destrezas fundamentales para un educador del siglo XXI. Estas habilidades y destrezas deseables en los estudiantes, responden a una serie de estrategias materializadas en las prácticas de aprendizaje y sobre todo, explicitadas en la forma en cómo a luz de este mapa de prácticas se evalúa el progreso alcanzado por los alumnos y su seguimiento. En este sentido, la evaluación de los aprendizajes debe valorarse tomando en consideración los siguientes puntos:

- La evaluación de los aprendizajes en los cursos facilita la determinación del nivel de progreso alcanzado por los educandos en cuanto a: contenido, habilidades de auto aprendizaje y habilidades para el trabajo colaborativo.

- La evaluación es consistente con las prácticas de aprendizaje.

- La evaluación es consistente con un enfoque alternativo, brinda espacios para retroalimentar a los educandos sobre sus avances, logros y debilidades, está enfocada a un mejoramiento continuo y no a un resultado absoluto.

- La evaluación contempla aspectos tanto cualitativos como cuantitativos.

- Los estudiantes conocen de manera precisa los aspectos en los cuáles serán evaluados en todas las actividades realizadas en los cursos.

El coordinador general de los cursos, se encargará de colaborar con su grupo de docentes para ir transformando poco a poco la fuerte tendencia institucional en el contexto de la Universidad Nacional de Costa Rica, donde se ha mantenido durante años, una clara tradición positivista.

\section{Infraestructura institucional}

La infraestructura y la compra de equipo audiovisual constituyen para este proyecto un reto muy importante. La Universidad Nacional de Costa Rica 
requiere crear espacios académicos apropiados para los estudiantes que cursan materias en una modalidad bimodal o virtual. Aún los esfuerzos realizados a nivel institucional son insuficientes para cubrir con una demanda que se ha delegado al alumno, obligándolo a invertir recursos por cuenta personal, que garantizan su acceso a las tareas virtuales asignadas en estos programas.

¡Esto no debe continuar así!, la institución tiene a su cargo la responsabilidad de tomar parte en este aspecto. Se espera que los informes de autoevaluación arrojados a la luz de este proceso, sirvan para crear la presión institucional necesaria, que en un mediano y largo plazo hagan tomar conciencia a las autoridades universitarias sobre esta problemática. Bajo esta perspectiva, la evaluación de la infraestructura institucional se fundamentará en:

- Identificar las carencias en cuanto al uso de laboratorios de acuerdo a la percepción docente y estudiantil.

- Identificar las carencias en la División de Educología de la UNA, con relación al equipo audiovisual y computacional disponible.

- Identificar las principales carencias de infraestructura y equipo que representan los mayores obstáculos para crear condiciones de calidad en este proyecto.

Muchos de los aspectos anteriormente citados ya son conocidos dentro de la cultura institucional y en la práctica educativa de los docentes; sin embargo, nos parece esencial sistematizarlo mediante la aplicación de instrumentos que recojan la percepción de los agentes involucrados.

\section{Seguimiento}

El seguimiento del proyecto será ejecutado por el coordinador general en colaboración con todos los profesores de las distintas cátedras de los cursos bimodales.

En este proyecto el seguimiento se concibe como un aspecto esencial para:

- Construir, reelaborar y seleccionar nuevos materiales didácticos.

- Solucionar a breve plazo problemas de contenido, tecnológicos o de relaciones humanas.

- Garantizar una práctica educativa homogénea en cuanto al enfoque pedagógico implementado por los docentes.

- Proponer innovaciones relacionadas con las prácticas de aprendizaje y a la luz de estas nuevas propuestas definir el uso de otras herramientas tecnológicas.

- Diseñar de manera paulatina una serie de guías didácticas que constituyan una memoria escrita sobre los materiales básicos en los cuales se fundamentan los cursos, los aportes personales de cada docente en su disciplina 
y área específica (tanto en experiencias de aprendizaje como de evaluación) y ejemplos de recursos realizados por los mismos estudiantes.

Las estrategias que utilizará el coordinador se sustentarán en: un seminario permanente, un taller de reflexión pedagógica al finalizar cada período lectivo y una bitácora personal del trabajo realizado por cada uno de los docentes mediante un blog.

El seminario permanente será convocado por el coordinador de manera quincenal y en estos espacios de trabajo se procederá a:

- Introducir a los nuevos docentes en el enfoque pedagógico del curso.

- Proponer nuevos materiales didácticos de manera conjunta.

- Proponer nuevas prácticas de aprendizaje y el uso de otras tecnologías digitales o analógicas.

- Ofrecer a los docentes charlas formativas en aspectos tecnológicos y de tutoría virtual (por parte del departamento de UNA Virtual).

- Elaborar en conjunto y durante cada semestre guías didácticas digitales con material didáctico y experiencias de aprendizaje probadas por los mismos docentes en su práctica profesional.

- Ofrecer espacios para que los estudiantes de distintas disciplinas puedan compartir con otros profesores sus realizaciones creativas (esto permitirá seleccionar de forma consensuada cuáles trabajos podrían ser incluidos en las guías didácticas).

Al finalizar cada semestre el coordinador general convocará a un taller de reflexión pedagógica con la intención de:

- Identificar fortalezas y debilidades en:

- La propuesta pedagógica de los cursos.

- Las prácticas de aprendizaje y de evaluación.

- Los materiales de estudio ofrecidos a los estudiantes.

- El equipo audiovisual y computacional disponible y sus horarios de uso.

- Identificar las fortalezas y requerimientos de la plataforma institucional Moodle.

- Definir las necesidades sentidas por el grupo de docentes colaboradores y a la luz de estos resultados la puesta en marcha de futuros procesos de capacitación.

- Brindar un informe sobre el trabajo realizado por la coordinación durante el período y los procesos de investigación encausados en torno al proyecto.

- $\quad$ Ofrecer la oportunidad para el diálogo abierto sobre el trabajo realizado por todos y todas. 
Finalmente el coordinador general abrirá un espacio privado a cada uno de los docentes de los distintos cursos, quienes tendrán la responsabilidad de moderar semanalmente durante todo el semestre un blog, cuyos únicos invitados serán los colegas de la cátedra. El blog conformará una bitácora personal de los profesores sobre sus experiencias semanales con sus estudiantes ante: cada uno de los contenidos, las prácticas de aprendizaje asignadas, las experiencias de evaluación, los materiales utilizados, los materiales desarrollados por los mismos estudiantes, las dificultades presentadas en la tutoría y las necesidades sentidas junto con sus posibles soluciones. El blog permitirá al grupo de profesores comunicar y socializar durante el proceso, aspectos tanto positivos como negativos que serán idealmente retroalimentados por todos de manera oportuna. Es reconocible en esta iniciativa, el efecto mariposa que podría ocasionar, pues si de previo el docente considera el blog como un medio de control por parte del coordinador general, perderá su sentido didáctico y colaborativo.

\section{Conclusiones}

Se han propuesto en este documento las bases de las cuales dependerá la exitosa transición en cuanto al uso e implementación de las tecnologías de la información y la comunicación para el aprendizaje virtual, en el contexto de la División de Educología de la Universidad Nacional de Costa Rica. Estas bases son el resultado de una experiencia docente y de investigación de varios años, que podrían resultar de interés a otras instituciones que se encuentren en el mismo marco de transición. Las políticas de uso e implementación de TIC's expuestas constituyen insumos ante los cuales otras instituciones $u$ organizaciones podrían tomar importantes decisiones para la actualización de sus currículum, perfiles docentes y manejo administrativo de sus cursos virtuales o bimodales.

\section{Referencias Bibliográficas}

- Becerra, M., Bavio, E. y otros (s.f.) Comisión asesora de educación a distancia. Informe final. Argentina, Ministerio de Educación, Ciencia y Tecnología.

- División de Educología (2008) Misión y Visión de la Unidad Académica. Documento recuperado de [http://www.una.ac.cr/cide/Educologia/index.html], la fecha [5 noviembre 2008].

- Duart, J. y Sangrà, A. (2000) Aprender en la virtualidad. España, Gedisa.

- Espiro, S. (2008) Aprendizaje. Especialización en Entornos Virtuales de Aprendizaje. Virtual educa.

- $\quad$ Espiro, S. (2008) Aprendizaje adulto. Especialización en Entornos Virtuales de Aprendizaje. Virtual educa. 
- García, A. (1998) "Indicadores para la evaluación de la enseñanza en una universidad a distancia", en Revista Iberoamericana de Educación a Distancia, No1, pp. 63-85.

- Juarez, A. y Assad, C. (2001) "Gestión y evaluación de un sistema a distancia", en Red de Educación a Distancia de las Universidades Nacionales Región Centro-Oeste, №1, pp. 14-18.

- $\quad$ Prieto, D. (2008) Planificar para construir futuro. Especialización en Entornos Virtuales de Aprendizaje. Virtual educa.

- $\quad$ Prieto, D. (2008) Fase de planificación operativa. Especialización en Entornos Virtuales de Aprendizaje. Virtual educa.

- Prieto, D. (2008) Evaluación y seguimiento. Especialización en Entornos Virtuales de Aprendizaje. Virtual educa.

- Prieto, D. y Van de Pol, P. (2006) E-learning comunicación y educación. El diálogo continúa en el ciberespacio. Costa Rica, Radio Nederland Training Centre.

- Vílchez, E. y Ulate, G. (2008) "Curso Recursos Didácticos para el Aprendizaje. Una experiencia en la Virtualidad”, en Revista Cognición, N 14, pp. 52-80.

- UNA. (2008) Modelo Pedagógico Institucional. Documento recuperado de [http://www.una.ac.cr], la fecha [5 noviembre 2008]. 